\title{
Tonotopic Distribution of Short-Term Adaptation Properties in the Cochlear Nerve of Normal and Acoustically Overexposed Chicks
}

\author{
Mark A. Crumling ${ }^{1,2}$ and James C. Saunders ${ }^{2}$ \\ ${ }^{1}$ David Mahoney Institute of Neurological Sciences, University of Pennsylvania, Philadelphia, PA 19104, USA \\ ${ }^{2}$ Department of Otorhinolaryngology: Head and Neck Surgery, University of Pennsylvania, Philadelphia, PA 19104, USA
}

Received: 14 February 2006; Accepted: 18 October 2006; Online publication: 3 January 2007

\begin{abstract}
Cochlear nerve adaptation is thought to result, at least partially, from the depletion of neurotransmitter stores in hair cells. Recently, neurotransmitter vesicle pools have been identified in chick tall hair cells that might play a role in adaptation. In order to understand better the relationship between adaptation and neurotransmitter release dynamics, short-term adaptation was characterized by using peristimulus time histograms of single-unit activity in the chick cochlear nerve. The adaptation function resulting from $100-\mathrm{ms}$ pure tone stimuli presented at the characteristic frequency, $+20 \mathrm{~dB}$ relative to threshold, was well described as a single exponential decay process with an average time constant of $18.6 \pm 0.8 \mathrm{~ms}$ (mean \pm SEM). The number of spikes contributed by the adapting part of the response increased tonotopically for characteristic frequencies up to $\sim 0.8 \mathrm{kHz}$. Comparison of the adaptation data with known physiological and anatomical hair cell properties suggests that depletion of the readily releasable pool is the basis of short-term adaptation in the chick. With this idea in mind, short-term adaptation was used as a proxy for assessing tall hair cell synaptic function following intense acoustic stimulation. After $48 \mathrm{~h}$ of exposure to an intense pure tone, the time constant of short-term adaptation was unaltered,
\end{abstract}

Correspondence to: Mark A. Crumling • MSRB III, Rm 9301, 1150 W. Medical Center Dr., Ann Arbor, MI 48109-0648, USA. Telephone: +1-734-7639680; fax: +1-734-6158111; email: crumling@umich.edu whereas the number of spikes in the adapting component was increased at characteristic frequencies at and above the exposure frequency. These data suggest that the rate of readily releasable pool emptying is unaltered, but the neurotransmitter content of the pool is increased, by exposure to intense sound. The results imply that an increase in readily releasable pool size might be a compensatory mechanism ensuring the strength of the hair cell afferent synapse in the face of ongoing acoustic stress.

Keywords: hair cell, single unit, auditory, exocytosis, synapse, afferent

\section{INTRODUCTION}

Primary auditory neurons generally show a vigorous response at the onset of acoustic stimuli, with the response then decaying over time, eventually reaching a steady firing rate (Kiang 1965; Westerman and Smith 1984; Javel 1996). This adaptation to a steady-state level occurs in stages, which in the literature are grouped into four classes based on the time constant $(\tau)$ : rapid $(\tau \sim 1-10 \mathrm{~ms})$, short-term $(\tau \sim 10-100 \mathrm{~ms})$, long-term $(\tau \sim 1-10 \mathrm{~s})$, and very long-term $(\tau \sim 10-$ $240 \mathrm{~s}$ ) (Harris and Dallos 1979; Westerman and Smith 1984; Javel 1996). Adaptation models have invoked a series of depletable vesicle "reservoirs" in hair cells to account for the stages (Schwid and Geisler 1982; Smith and Brachman 1982; Geisler and Greenberg 1986; Westerman and Smith 1988). This depletion hypothesis is supported by work on goldfish saccular 
afferents where excitatory postsynaptic potentials (EPSPs) decrease in amplitude during a constant acoustic stimulus (Furukawa et al. 1978; Furukawa and Matsuura 1978).

Functional vesicle pools have been discovered in hair cells that could be involved in adaptation. Using membrane capacitance to monitor exocytosis, hair cells of the mouse (Moser and Beutner 2000) and chicken (Eisen et al. 2004; Spassova et al. 2004) have been found to undergo vesicle fusion in at least two phases: a "readily releasable" pool undergoes exocytosis first and depletes with a time constant ranging from 10 to no more than $50 \mathrm{~ms}$, depending on the species (Moser and Beutner 2000; Spassova et al. 2004); this wave of exocytosis is followed by the fusion of a "reserve" pool of vesicles that lasts for at least one second in vitro (Moser and Beutner 2000; Eisen et al. 2004).

The anatomical basis for these pools may lie in a synaptic specialization called the dense body, a form of synaptic ribbon. This structure seems to hold vesicles in close proximity to a release site (Smith and Sjostrand 1961; Martinez-Dunst et al. 1997; Lenzi et al. 1999). There is general agreement between the number of vesicles tethered to dense bodies and the number in the readily releasable pool (Spassova et al. 2004). Vesicles are also located in the cytoplasm, apparently unassociated with dense bodies. Therefore, the readily releasable pool may be composed of vesicles tethered to dense bodies, and the reserve pool may correspond to the cytoplasmic vesicles. There is a tonotopic gradient in the size of dense bodies in the chick basilar papilla, endowing tall hair cells toward the base (high frequency end) of the papilla with more dense-body associated vesicles and, presumably, larger readily releasable pools than those at the apex (low frequency end) (MartinezDunst et al. 1997).

The relationship between adaptation and vesicle pool depletion is poorly understood. Pool kinetics have been described for chick tall hair cells, the primary afferent-bearing receptor cells of the basilar papilla. While the exact kinetics have yet to be determined, the time constant of readily releasable pool depletion was found to be less than $50 \mathrm{~ms}$ (Spassova et al. 2004). In the same study, short-term adaptation in cochlear nerve afferents was shown to occur with a time constant of about $20 \mathrm{~ms}$ and recovery from adaptation had kinetics similar to refilling of the readily releasable pool. Consequently, the hypothesis was offered that depletion of the readily releasable pool was the basis of short-term adaptation. Here we test this hypothesis further by characterizing short-term adaptation with respect to characteristic frequency $(\mathrm{CF})$ in single units of the normal and acoustically overexposed chick cochlear nerve, with the aim of comparing adaptation properties to known anatomical and physiological properties of chick hair cells.

\section{METHODS}

\section{Animal preparation}

White Leghorn chicks (Gallus domesticus) between 6 and 22 days of age were anesthetized with an intramuscular injection of a $25 \%$ ethyl carbamate (Urethane) solution ( $0.1 \mathrm{ml}$ per $10 \mathrm{~g}$ of body weight). A tracheotomy was performed to allow free breathing, and the dorsal and left posterior-lateral regions of the skull were exposed. The soft tissue around the ear canal was excised to expose the tympanic membrane. The skull was secured to a head holder with ethyl cyanoacrylate glue and dental acrylic, and a 5-mm diameter hole was opened over the posterior-lateral portion of the skull. The hole permitted access to the inner bony layer of the temporal bone. Access to the recessus scala tympani was achieved via a second hole $(\sim 1.5 \mathrm{~mm}$ in diameter $)$ made through this inner layer. The membranous lining of the cochlear capsule was pierced, and the cochlear ganglion, when viewed through this access hole, was recognized as a white band on the medial wall of scala tympani. This preparation is similar to that used in Saunders et al. 1996b.

Each chick was tested in an acoustically attenuated chamber. The head holder was firmly secured to a frame that held the body in a horizontal orientation. The body was placed on a thermostatically controlled heating pad set to $41^{\circ} \mathrm{C}$. For the data presented here, the head temperature was monitored in the mouth and maintained at $\sim 41^{\circ} \mathrm{C}$ by a DC halogen lamp adjacent to the head. A subset of these data was also included in the "warm" temperature group in a study of temperature effects on cochlear nerve activity (Crumling and Saunders 2005).

\section{Acoustic stimulation}

The ear was stimulated with a closed-field sound system consisting of an earphone (Model DT-48; Beyer Dynamic, Hicksville, NY, USA) and an attached sound tube (10 cm long, $5 \mathrm{~mm}$ in diameter). A 0.5$\mathrm{mm}$ probe tube connected to a microphone (Model ER-7; Etymotic Research, Elk Grove Village, IL, USA) was placed down the center of the sound tube, and the sound tube was sealed around the tympanic membrane. Output from the probe-tube microphone was connected to the analyzer section of a frequency synthesizer (System One; Audio Precision, Beverton, OR, USA). The generator section of the synthesizer produced tonal stimuli under computer control. For 
calibration of the speaker, the computer converted the voltage values from the probe-tube microphone to $\mathrm{dB}$ SPL (relative to $20 \mu \mathrm{Pa}$ ) using the microphone calibration constant. For each of the test frequencies, an automatic procedure adjusted the voltage output of the synthesizer to produce $100 \mathrm{~dB}$ SPL. Different levels of stimulation were achieved under program control by modifying the synthesizer voltage output relative to the $100 \mathrm{~dB}$ SPL value to achieve the desired SPL.

\section{Single-unit recordings}

Borosilicate glass microelectrodes (15-50 M , filled with $3 \mathrm{M} \mathrm{KCl}$ ) were secured to a microdriver and advanced into scala tympani toward the cochlear nerve. Electrical signals were amplified and sent to an oscilloscope, audio monitor, and level detector for the determination of spike timing. The timing of nerve spikes was recorded with $0.1 \mathrm{~ms}$ resolution by a personal computer. The data were stored on hard disk, and only data from well-isolated, stable responding units were logged.

\section{Recording protocol}

The search stimulus consisted of a burst of band-limited noise $(0.1-5.0 \mathrm{kHz}, 40-\mathrm{ms}$ in duration, 2.5 or $5 \mathrm{~ms}$ rise/ decay time), presented at a rate of $9 / \mathrm{s}$. When an acoustically driven unit was encountered by the microelectrode, spontaneous activity was determined from a 6-s sample of activity in the absence of intentional acoustic stimulation. The stimulus was then switched to a tone burst with the same temporal parameters as the search stimulus, and the receptive field of the unit was determined by constructing a spectral response plot (similar to Kaltenbach and Saunders 1987; Saunders et al. 1996b). The perimeter of the receptive field represented the tuning curve of the unit and was defined as a visually noticeable difference from spontaneous activity. The CF and threshold at CF (in dB SPL) were determined from the tuning curve.

For each unit, the peristimulus time (PST) response was examined at the CF by using bursts of $100-\mathrm{ms}$ duration (2.5 or $5 \mathrm{~ms}$ rise/decay time) at a level of 20 $\mathrm{dB}$ above threshold. PST histograms were generated by summing the discharge activity from a total of 376 tone bursts delivered $166 \mathrm{~ms}$ apart. This interstimulus interval allowed for recovery from adaptation (Spassova et al. 2004). The phase-angle of each tone burst was random with respect to the burst onset. In some cases, PST histograms were also collected above or below the $\mathrm{CF}$ to investigate the effect of stimulus frequency. In these instances, the level of the stimulus was set at $\sim 20$ $\mathrm{dB}$ above the threshold at that frequency as defined by the tuning curve. This method of stimulus stan- dardization for off-CF frequencies was chosen over another possible procedure where stimulus levels were selected that produced the same firing rate. This "isoresponse" procedure required too much time to complete while maintaining contact with the unit to be of practical value. To investigate the effect of stimulus level, some histograms were collected at the $\mathrm{CF}$ at a level of $100 \mathrm{~dB}$ SPL.

\section{Acoustic overexposure}

Chicks age 5-8 days were placed individually into one of six chambers of a circular wire mesh cage housed in an acoustically attenuated chamber. Each chick was supplied with enough food and water for 2 days. The cage was positioned beneath a 30-cm loudspeaker (Model E120, JBL, Inc.) and a 0.9-kHz tone at $120 \mathrm{~dB}$ SPL was played for $48 \mathrm{~h}$. After the exposure, the birds were surgically prepared and recordings were initiated. Agematched, unexposed birds served as controls.

\section{Data analysis}

Data are presented from a total of 209 units from 57 birds. Of those totals, 36 unexposed birds provided 141
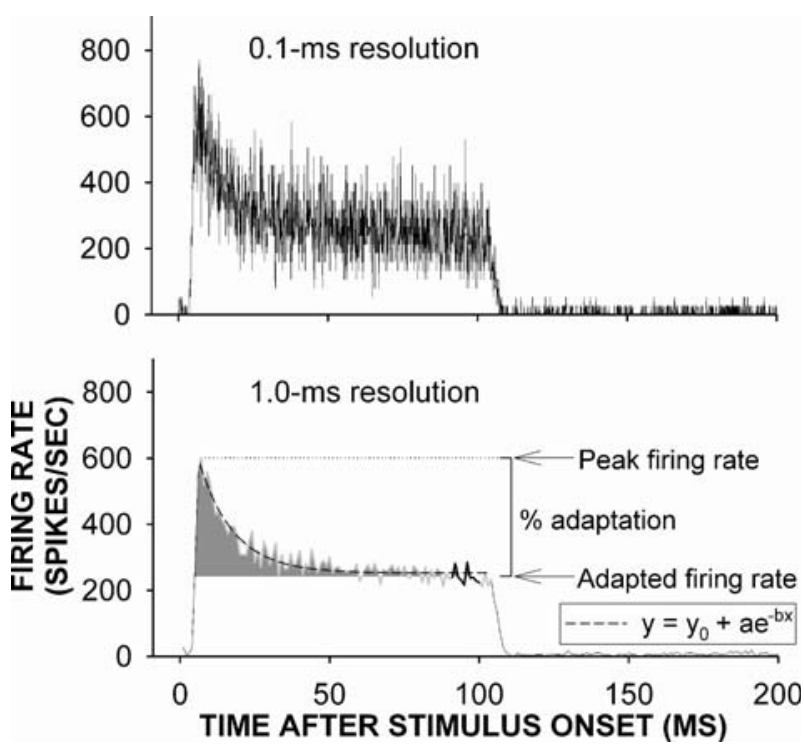

FIG. 1. Analysis of PST histograms. The raw data were collected with a sampling resolution of $0.1 \mathrm{~ms}$ (upper plot). The histograms were re-binned at 1.0-ms resolution to decrease the local variability (lower plot). Parameters describing the PST response at $1.0-\mathrm{ms}$ resolution are defined as follows: peak firing rate $=$ maximum driven firing rate; adapted firing rate $=$ average firing rate during the $91 \mathrm{st}$ through 100th 1-ms bins after stimulus onset (blackened portion of the gray curve); percent adaptation = percent change from the peak to adapted firing rate; phasic component (dark gray area) = number of spikes in the adapting part of the curve. The single exponential fit [Eq. (1), dashed line] of the adaptation function (gray curve) provided the adaptation time constant, as well as the predicted final adapted rate $\left(y_{0}\right)$ for this stage of adaptation. 


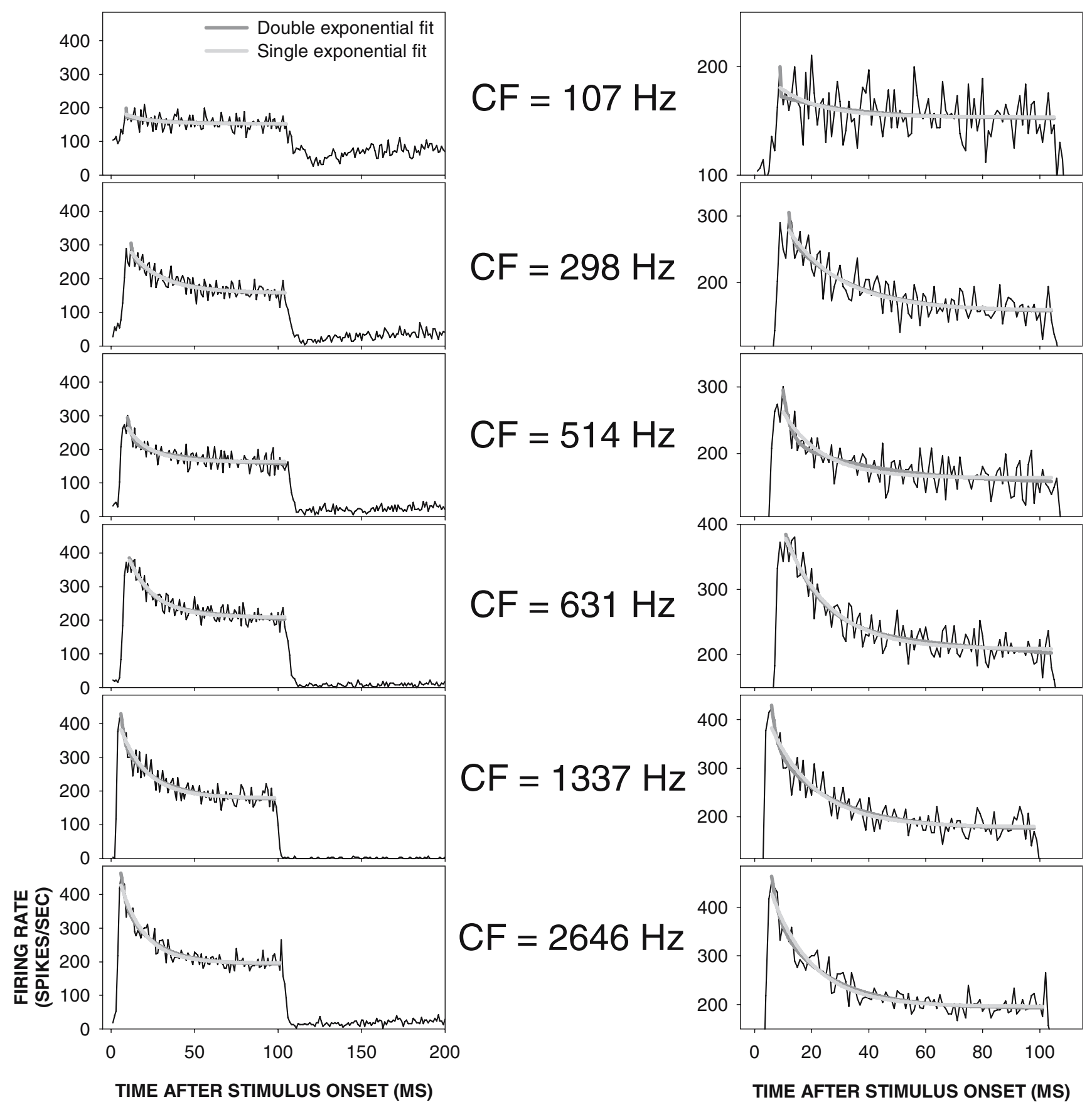

FIG. 2. Example PST histograms and adaptation curve fits. Left-hand panels show an example histogram from each CF analysis bin for normal ears described in Methods. Right-hand panels show the adapting portion of the same histograms in greater detail. The adaptation function of each PST histogram is fit with both single and double exponentials [Eqs. (1) and (2)]. The adaptation functions were well described by the single exponential equation.

units, and 21 acoustically overexposed birds provided 68 units. Of the unexposed animals, 8 birds provided 14 units for the stimulus level manipulations, 10 birds provided 26 units for the stimulus frequency manipulations, and 20 birds provided 81 units for the agematched control group of the acoustic overexposure experiments. The sample size $(n)$ presented in the figures represents the number of units.
The analysis of PST histograms is described in Figure 1. To minimize local variations in the PST histograms, the raw data were re-binned at a resolution of Histogram data are presented as spikes/s for each bin. The time course of adaptation was analyzed by using the data beginning with the initial peak response of the PST histogram and ending just before the falling phase of the stimulus envelope. These 
adaptation curves were fitted with a single or double exponential decay function [Eqs. (1) and (2)], each containing an offset term, using the nonlinear regression capability of a commercially available graphing package (Sigma Plot).

$$
\begin{gathered}
y=y_{0}+\mathrm{ae}^{-b x} \\
y=y_{0}+\mathrm{ae}^{-b x}+\mathrm{ce}^{-d x}
\end{gathered}
$$

The variable $y$ in Eqs. (1) and (2) is the firing rate at any given time, $x ; y_{0}$ is the offset term; $b$ and $d$ are inverses of the time constants. The following additional parameters were taken directly from the histograms: peak firing rate was the maximum rate of the onset response at 1-ms resolution; adapted firing rate was the average rate occurring in the 91st through 100th 1-ms bins after the onset of the stimulus; the phasic component of the response was calculated by summing the histogram spikes that occurred in excess of the adapted firing rate at 1-ms resolution.

To assess gradients in the above parameters across CFs, the data from all 36 unexposed birds (normal ears) were organized so that the CF range of the units was broken into six logarithmically spaced bins with center frequencies from 0.13 to $2.28 \mathrm{kHz}$ (each bin was 0.820 octaves wide). This allowed the utilization of a one-way analysis of variance (ANOVA) on the data in the CF bins to detect statistically significant variations in the parameters with $\mathrm{CF}$. In the analysis of the effects of acoustic overexposure, the exposed and age-matched control datasets were each organized into five $\mathrm{CF}$ bins with center frequencies from 0.14 to $2.29 \mathrm{kHz}$ (1.004 octave bin width) and the data in the bins were compared by using a two-way ANOVA to detect significant differences between control and exposed groups across the CF bins. When significant differences were detected by the two-way ANOVA, Tukey's test was performed to determine which CF bins contributed to the difference. When individual units were subjected to different experimental conditions (e.g., different stimulus levels), paired $t$-tests on the means for each condition were used to detect significant differences. The criterion for statistical significance in all cases was $p<0.05$.

\section{RESULTS}

Tonotopic variations in short-term adaptation in the normal ear

The response at stimulus onset differed between low and high CF units. Low-frequency units adapted little compared to high CF units during 100-ms test tones presented $20 \mathrm{~dB}$ above threshold at the CF.

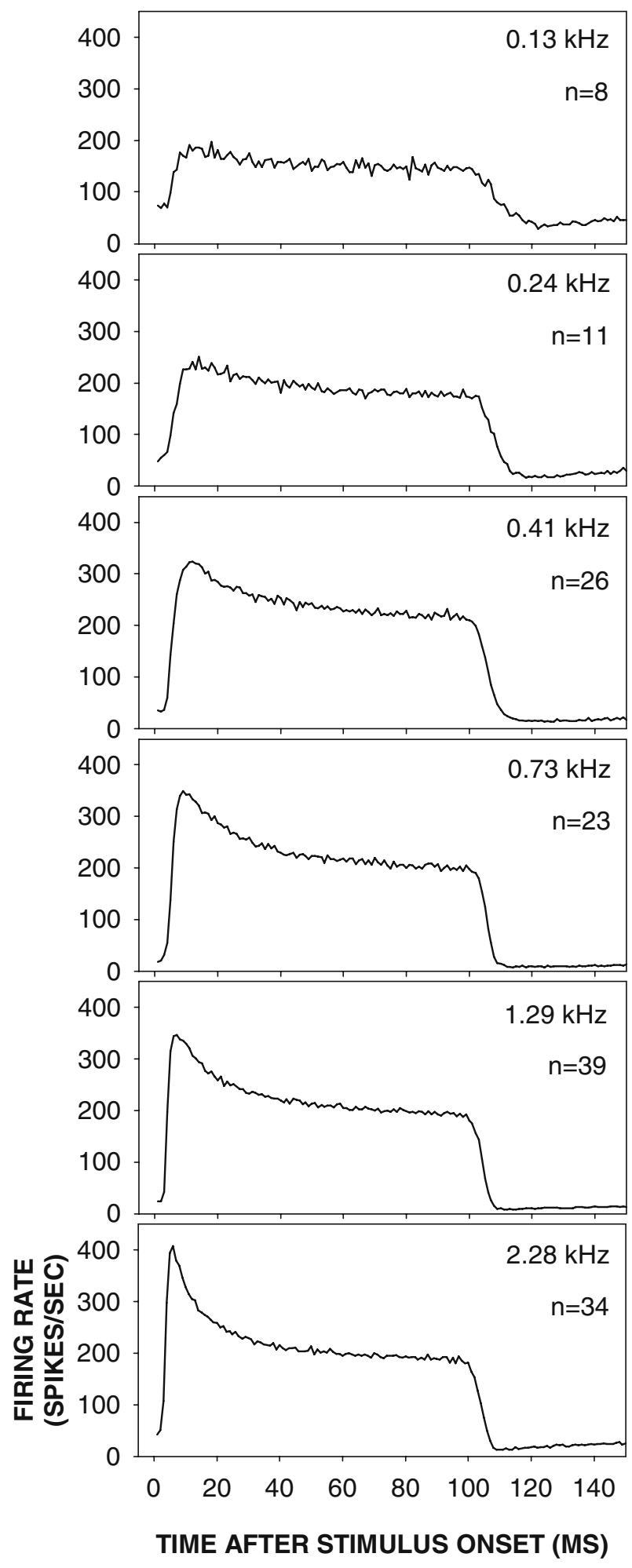

FIG. 3. Averaged PST histograms. The PST histograms analyzed for each of the CF bins were averaged to generate composite histograms for each frequency range. The center frequency of the CF bin is shown for each plot. High CF units appeared to have larger, more quickly adapting onset responses than units with low CFs. 

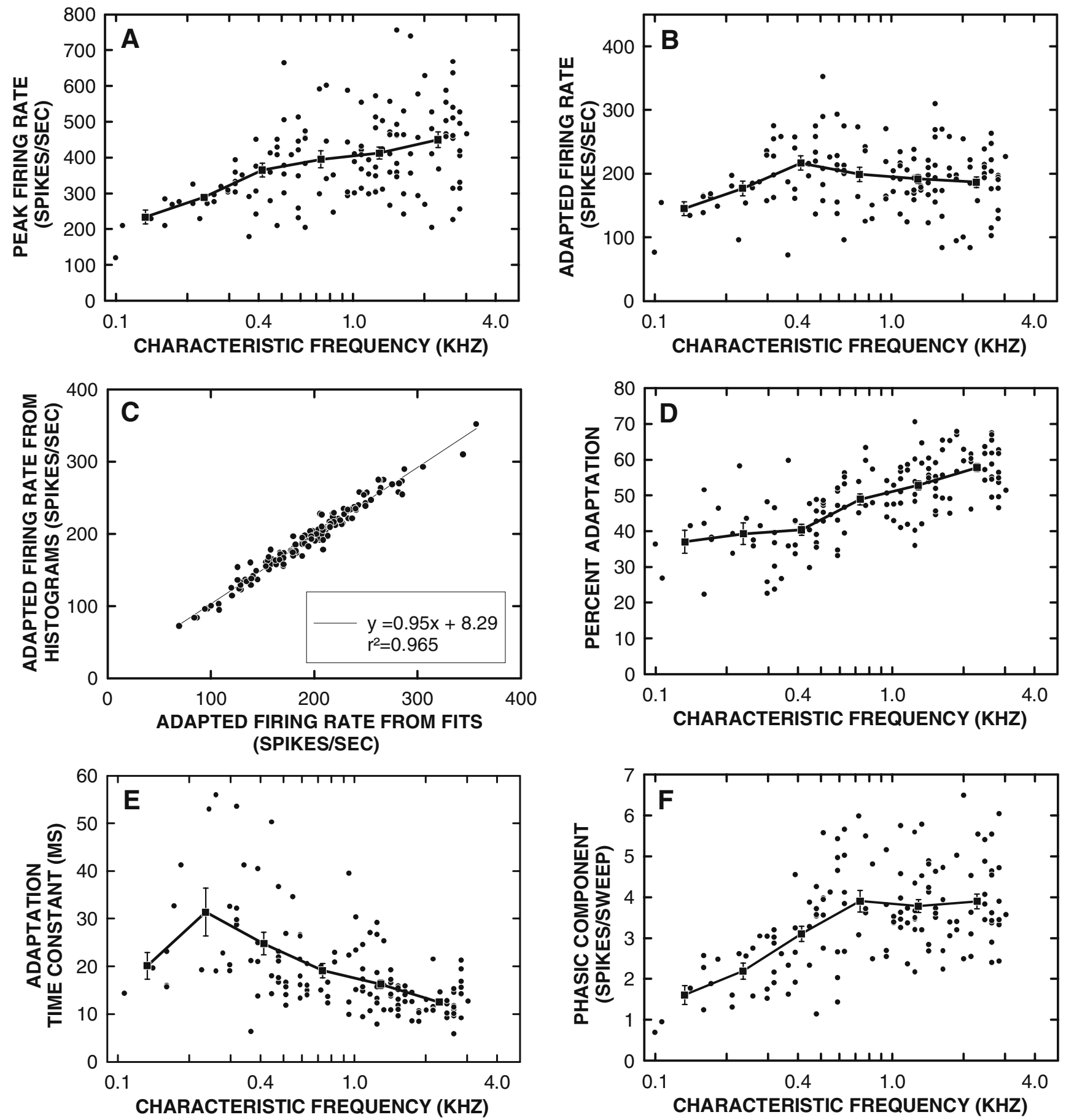

FIG. 4. Analysis of PST histogram parameters. Circles represent data for individual units, whereas the squares with error bars (SEM) are the means for the same data when grouped into the CF bins. (A) Units showed a progressive increase in the peak firing rate across CF ( $p<0.001$ ). (B) The adapted firing rates were more constant (only the $0.41-\mathrm{kHz}$ bin was significantly different from the $0.13-\mathrm{kHz}$ bin). (C) The adapted rates directly measured from the histograms showed a near one-to-one relationship (linear regression slope $=0.95$ ) with the predicted asymptotic rates from single exponential fits, indicating that this stage of adaptation is complete by the end of the 100-ms stimulus. (D) Percent adaptation was greatest in high CF units ( $p<0.001$ ). (E) Units with higher CFs generally adapted more quickly $(p<0.001)$. Time constants were in the range of those classified as "short-term" adaptation in mammals. (F) The number of spikes in the phasic component of the response grew up to the $0.73-\mathrm{kHz} C F$ bin $(p<0.001)$. Above this bin, the phasic component remained constant. ( $n \geq 137$ for each scatter plot.) 

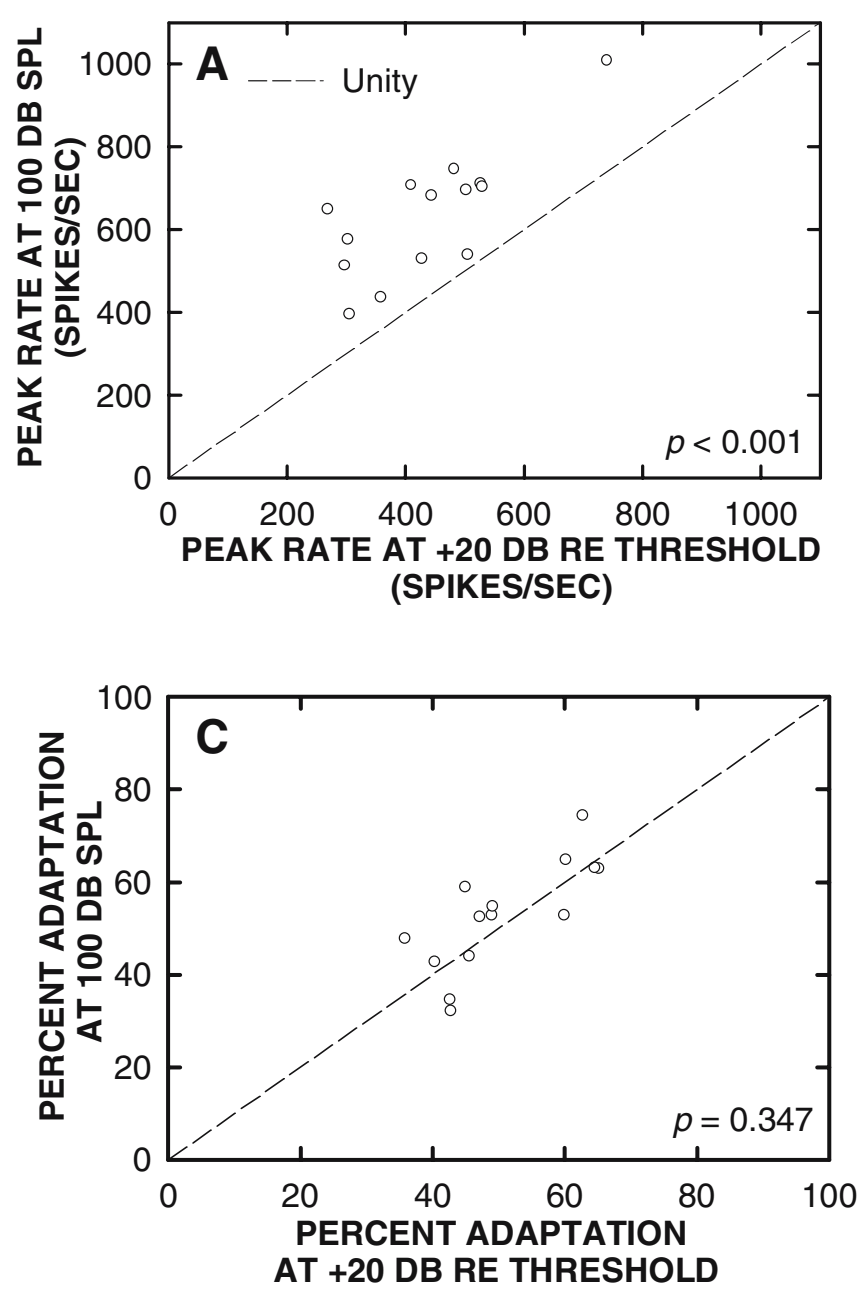
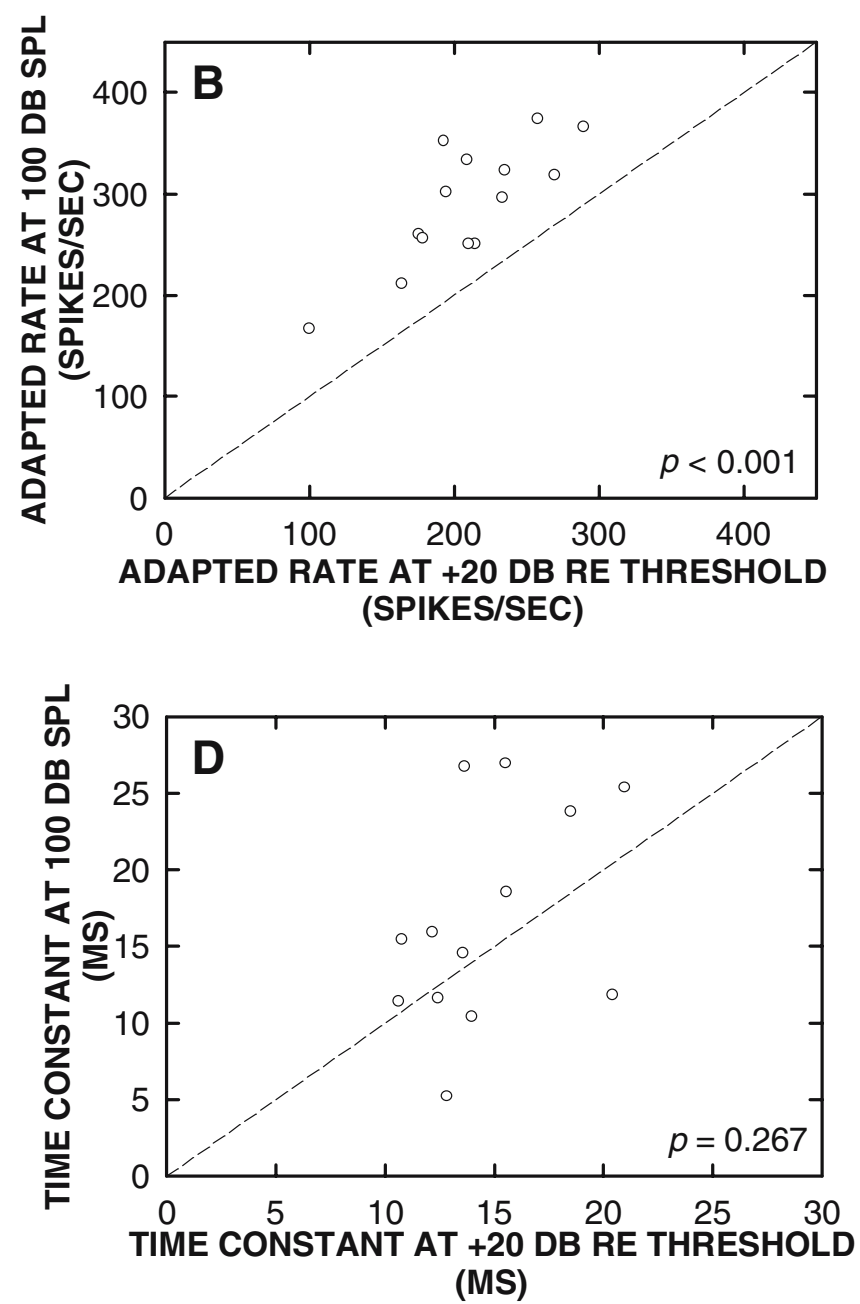

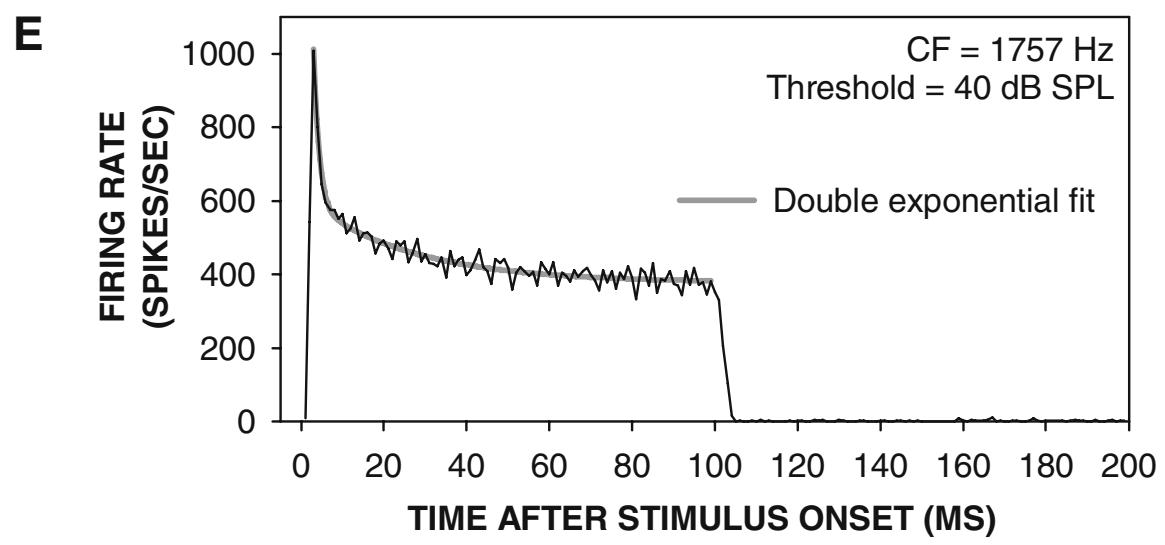

FIG. 5. Summary of stimulus level manipulation. Peak (A) and adapted (B) rates were increased at the higher stimulus level, but the percent adaptation (C) and time constant (D) were stable. (E) Histogram collected at $100 \mathrm{~dB}$ SPL where the adaptation function was better described as a double exponential decay process. This histogram was excluded from the analysis of time constant in D. $(n=14$ for A, B, and C; $n=13$ for D.)

This tendency is readily apparent in individual PST histograms (Fig. 2) and in averaged PST histograms (Fig. 3) from units collected in different CF ranges (CF bins in Methods). An exaggerated peak at the response onset is clearly seen for units with high CFs.
To quantify the changes in the adapting response across CFs, individual parameters of the PST histograms were examined. Peak firing rates in response to $\mathrm{CF}$ tones presented $20 \mathrm{~dB}$ above threshold ranged from about 100 to almost 800 spikes per second and 


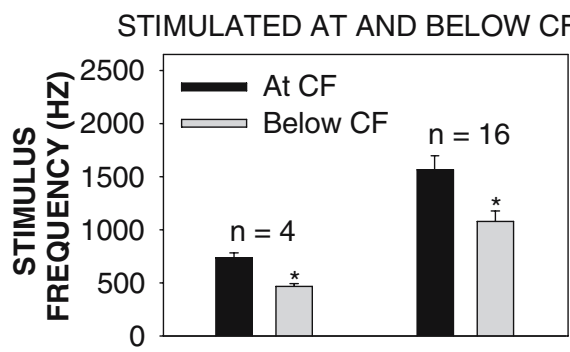

STIMULATED AT AND ABOVE CF
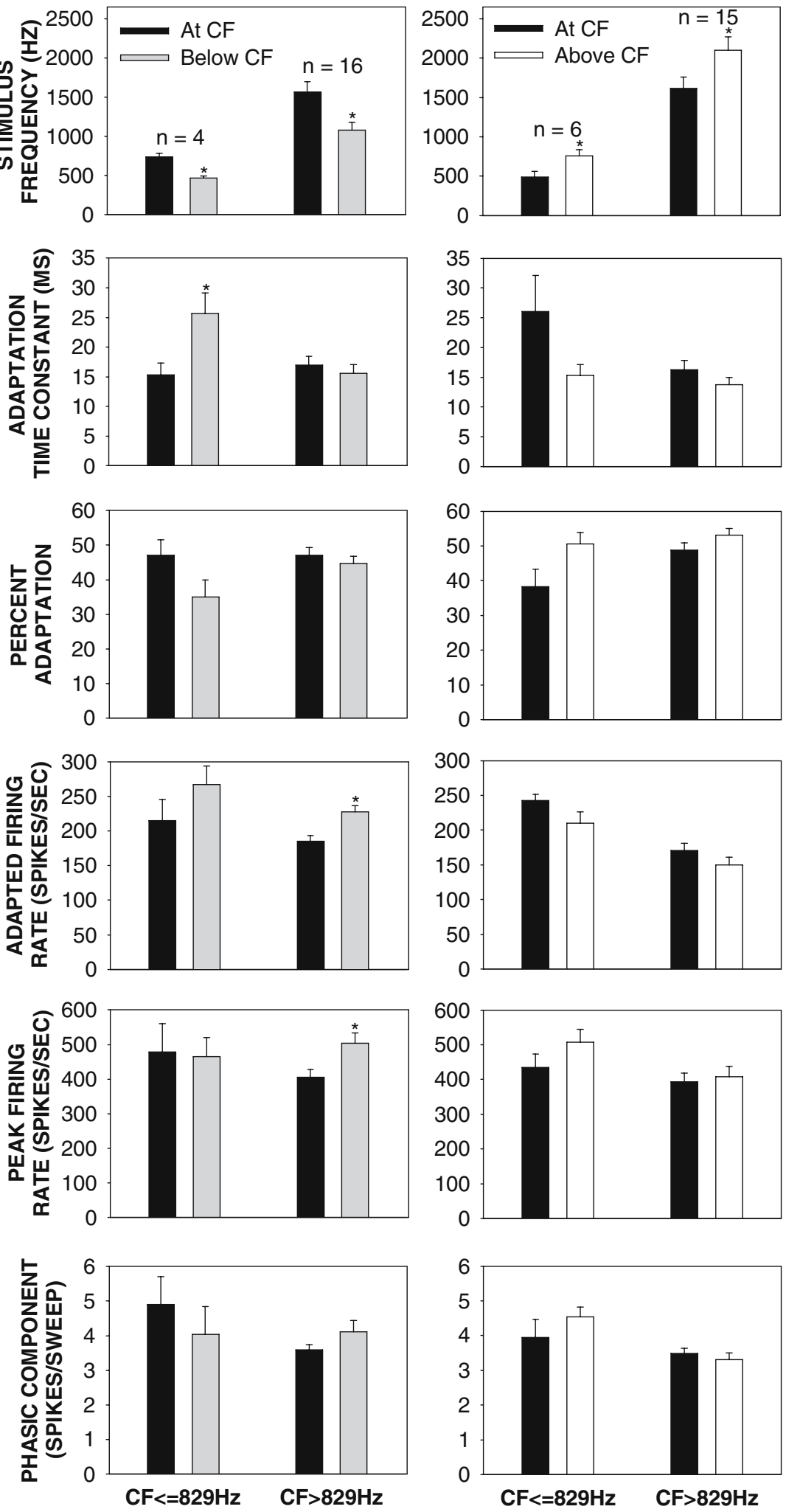

FIG. 6. Analysis of stimulus frequency effects on histogram parameters. Left-hand panels represent the data collected when units were stimulated both at and below the CF, whereas the right-hand panels represent the data collected when units were stimulated both at and above the CF. The data are broken into two CF ranges: CF $\leq 829 \mathrm{~Hz}$ and CF $>829 \mathrm{~Hz}$. Asterisk $\left({ }^{*}\right)$ denotes significantly different from the "at CF" condition within the same CF group. The adaptation time constant of units with an $829-\mathrm{Hz}$ or less CF was sensitive to stimulus frequency when stimulated below the CF. Peak and adapted firing rates of units with CFs above $829 \mathrm{~Hz}$ were sensitive to stimulation below the CF. 
were tonotopically graded, with the highest peak firing rates at the highest CFs (Fig. 4A). Across CFs, the firing rate decayed from the peak and settled to an average rate of about 150 to 200 spikes/s in each of the CF bins. The adapted level of activity showed no systematic gradation with CF (Fig. 4B). The graded nature of the peak response rate in contrast to the more constant nature of the adapted rate led to a progressive increase in percent adaptation with CF (Fig. 4D).

The decay from the peak to the adapted firing rate was well described as a single exponential decay process. Example single and double exponential fits using Eqs. (1) and (2) from Methods are shown in Figure 2. The fast component returned by the double exponential fits is comparable in amplitude to the histogram noise. Thus, there is little compelling evidence of a rapid adaptation component. This is in contrast to PST responses of mammalian auditory nerve fibers collected using stimuli of similar level and the same histogram bin width (1 ms). The mammalian adaptation functions so collected are well described by a double exponential with a rapid component rising well above the histogram noise (Westerman and Smith 1984, 1987).

The average time constant of the single exponential fits places this adaptation phenomenon in the "short-term" range, according to the classification scheme used in mammals (Harris and Dallos 1979; Westerman and Smith 1984, 1987). The average time constant for all units was $18.6 \pm 0.8 \mathrm{~ms}$ (mean $\pm \mathrm{SEM}$ ) and the range was 5.7-55.9 ms. A comparison of time constants in the CF bins reveals that the time constants are shortest at high CFs (Fig. 4E). Because of the noise in the PST histograms and the low peak amplitudes of the responses of the lowest CF cells, the fitting algorithm could not fit the adaptation time course of four units with CFs of $0.21 \mathrm{kHz}$ and lower. Therefore, no time constants are included for these units. Low-frequency cells were encountered rarely, so the lowest frequency bin in the time constant analysis contains rather few units $(n=6)$.

In addition to providing a time constant for the adaptation process, the single exponential fit also provides a prediction of the final adapted firing rate $\left(y_{0}\right)$ for this stage of adaptation. Across the population of units sampled, this predicted value closely mirrors the adapted firing rate measured directly from the PST histograms as the average rate occurring in the 91st through 100th 1-ms bins after the onset of the stimulus. When the $y_{0}$ values are plotted against the adapted rates measured directly from the histograms, a linear regression returns a slope that is near 1 (slope $=0.95$; Fig. 4C), indicating that the $100-\mathrm{ms}$ tone duration was sufficient to bring this stage of adaptation to completion.
The interaction of the peak and adapted firing rates with the adaptation time constant renders the onset of the response more pronounced at high, compared to low, CFs. At low CFs, the peak firing rates are low, and the difference between the peak and adapted firing rates is small. At high CFs, the peak rates are high, and there is a larger divergence between the peak and adapted rates. With their shorter adaptation time constants, the net result is an enhanced onset response in high $\mathrm{CF}$ units. In the chick basilar papilla, anatomical measurements have shown that dense body size increases with distance from the apical end (Martinez-Dunst et al. 1997). The presumed correlate of this is an increase in the size of readily releasable pool, which could be involved in the enhanced onset response. If the depletion hypothesis of adaptation is valid, the magnitude of the adapting portion of the response should also increase with CF. In order to assess this, the number of spikes contributed to each histogram by the adapting, or phasic, part of the response was obtained by summing those spikes that occurred in excess of the adapted firing rate. This area-under-the-curve measure should grow in proportion to the neurotransmitter pool that was emptied, assuming a linear relationship between released quantal content and spiking probability in the receiving afferent. The result of such an analysis is shown in Figure 4F. The number of spikes contributed to the histogram by the phasic component increased steadily with CF until approximately 0.7$0.8 \mathrm{kHz}$. At higher CFs, this value was constant.

\section{Effects of stimulus level and frequency}

By using a fixed stimulus level relative to threshold, apparent CF effects could be produced if the $+20-\mathrm{dB}$ stimulus systematically falls on a different portion of the rate-level function in units at different CFs. To rule out this concern, a portion of the units $(n=14)$ were stimulated both at $20 \mathrm{~dB}$ above threshold and at $100 \mathrm{~dB}$ SPL at the CF. The average $+20-\mathrm{dB}$ stimulus employed in these units had a level of $54.1 \pm 2.6 \mathrm{~dB}$ SPL, and the maximum was $73 \mathrm{~dB}$ SPL. Both peak and adapted firing rates were significantly elevated in units stimulated at $100 \mathrm{~dB}$ SPL compared to $+20 \mathrm{~dB}$ (Fig. 5A and B). The average peak rate in $100 \mathrm{~dB}$ SPL stimulated units was $634.9 \pm 41.1$ spikes/s compared to $435.8 \pm 33.9$ spikes $/ \mathrm{s}$ in the $+20-\mathrm{dB}$ condition $(p<0.001)$. Adapted rates were $290.0 \pm 16.1$ spikes $/ \mathrm{s}$ at $100 \mathrm{~dB}$ SPL and $208.7 \pm 12.9$ at $+20 \mathrm{~dB}(p<0.001)$. These rates were higher in all units when stimulated at $100 \mathrm{~dB}$ SPL. In contrast, percent adaptation (Fig. $5 \mathrm{C} ; 52.7 \pm 3.2 \%, 100 \mathrm{~dB}$ SPL; 50.7 $\pm 2.6 \%,+20 \mathrm{~dB}$; $p=0.347$ ) and the adaptation time constant (Fig. 5D; $16.1 \pm 1.9 \mathrm{~ms}, 100 \mathrm{~dB}$ SPL; $14.2 \pm 1.0 \mathrm{~ms}$, +20 dB; $p=0.267)$ were unaffected. Consequently, the stimulus 


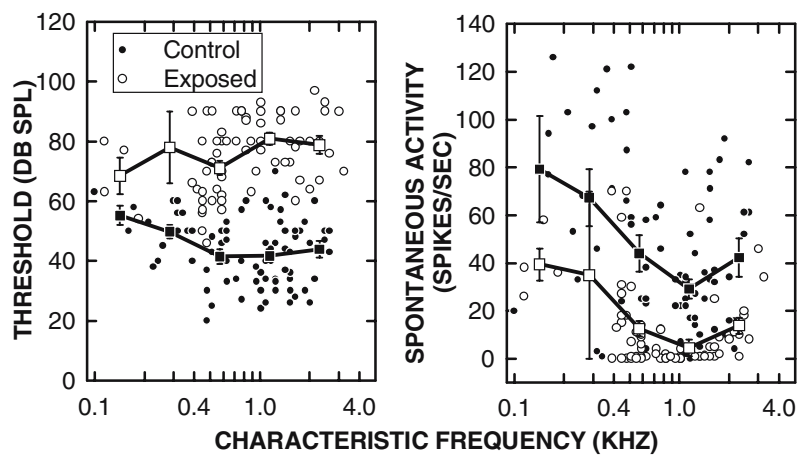

FIG. 7. Effect of acoustic overexposure on threshold and spontaneous activity. Exposure to the $0.9-\mathrm{kHz}, 120-\mathrm{dB}$ SPL tone for $48 \mathrm{~h}$ produced similar effects on threshold and spontaneous activity as those reported previously (Saunders et al. 1996b). PST histograms from these units were analyzed to determine the effects of acoustic overexposure on PST histogram parameters.

level appears to have little to do with the adaptation differences across CFs. Curiously, the adaptation function of one unit was better described as a double exponential when stimulated by the $100 \mathrm{~dB}$ SPL tone (Fig. 5E).

Because of the sharp tuning of cochlear nerve units in the chick and an absence of low-frequency tails like those of mammalian tuning curves, it was impossible to elicit sound-evoked discharge activity at the same frequency in every unit. As such, all of the preceding data were collected by stimulating units at their CF. This introduced the confounding variable of stimulus frequency when sampling at different CFs. Because the stimulus frequency covaried with the $\mathrm{CF}$ of the unit, it is possible that the changes seen across CFs were unrelated to physiological differences at distinct cochlear locations. Rather, the physiology at different CFs might be similar, but the adaptation function differs because it is sensitive to the acoustic frequency of the stimulus. To address this concern, additional PST histograms were collected for some units by using stimuli above and/or below the CF. Frequencies were chosen that were as far from the $\mathrm{CF}$ as possible with thresholds of $80 \mathrm{~dB}$ or less.

Figure 6 shows the data from the stimulus frequency manipulations broken into two $\mathrm{CF}$ ranges, using a $\mathrm{CF}$ of $829 \mathrm{~Hz}$ as the splitting point. This is close to the $\mathrm{CF}$ at which the phasic component began its plateau (Fig. 4F). The data indicate that with CFs of $829 \mathrm{~Hz}$ or lower, there was an effect of stimulus frequency on the adaptation time constant when units were stimulated below their CFs, compared to at their CFs. The time constant was longer when below-CF stimulation was used, an effect similar to the change with decreasing $\mathrm{CF}$. There were no significant differences detected in the phasic component when the stimulus frequency was altered, but the peak and adapted firing rates in the high CF group were elevated by stimulation below the $\mathrm{CF}$, which contrasts with the effect of decreasing CF. The data suggest that the adaptation time constant is sensitive to stimulus frequency, whereas the phasic component is insensitive. Thus the change in time constant with CF can be explained, at least in part, by covariance of $\mathrm{CF}$ and stimulus frequency.

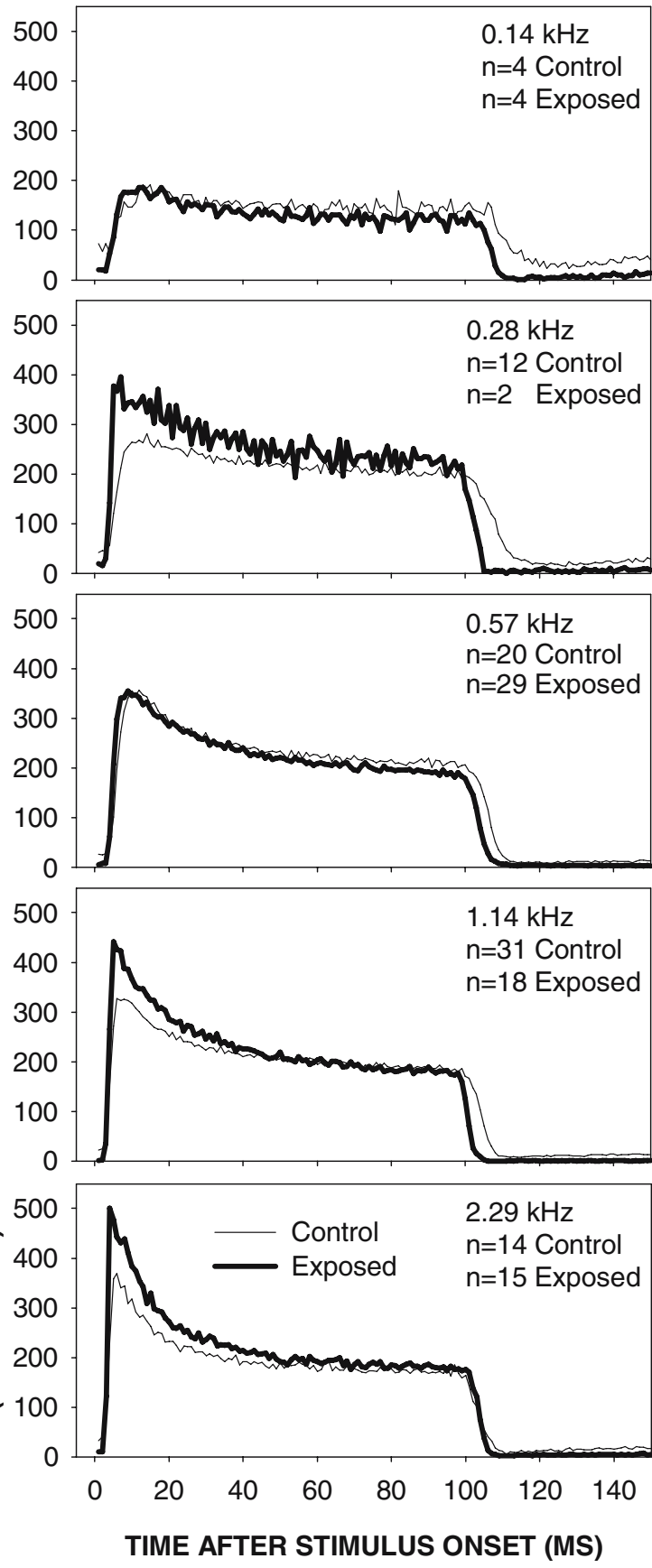

FIG. 8. Averaged PST histograms for the acoustic overexposure. When the histograms from each CF bin described in Methods for the acoustic overexposure experiment were averaged, an exaggerated onset response in exposed units was seen in comparison to agematched controls, particularly in units in the 1.14- and 2.29-kHz CF bins. Note the similar activity level to which control and exposed units adapted. The center frequency of the CF bin is shown for each plot. 

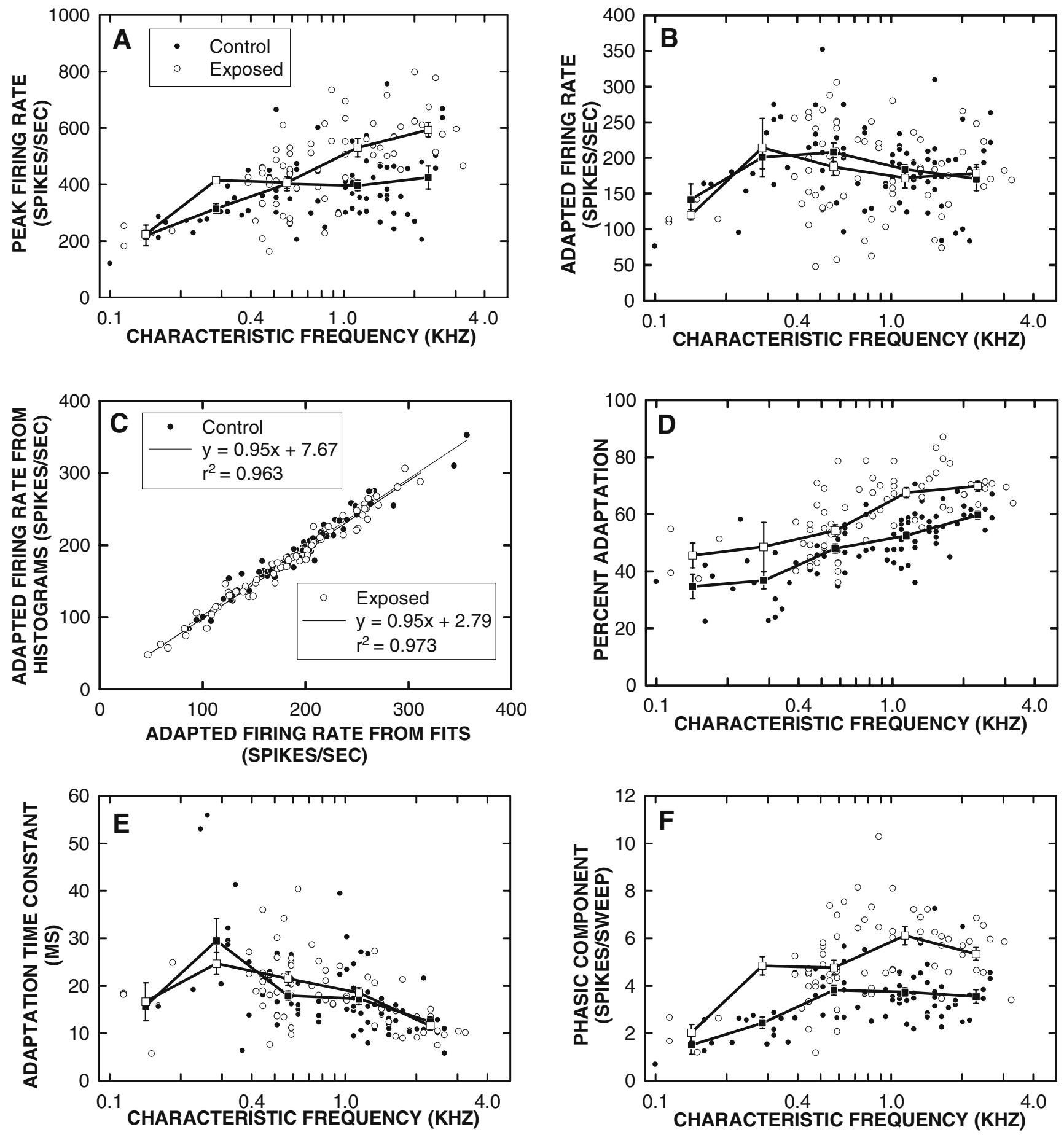

FIG. 9. Analysis of PST histogram parameters for the acoustic overexposure. (A) Peak firing rates were elevated after the exposure in the 1.14and $2.29-\mathrm{kHz}$ bins $(p<0.05)$. (B) The adapted firing rates were unchanged. (C) In both control and exposed units, the adapted rates directly measured from the histograms were comparable to the asymptotic rates predicted from single exponential fits (linear regression slopes $=0.95$ for both conditions), indicating that the short-term adaptation stage was complete by the end of the stimulus for both groups. (D) Percent adaptation was increased in the three highest CF bins $(p<0.05)$. (E) The short-term adaptation time constant was unaltered by the exposure. $(F)$ The phasic component of the response was significantly larger $(p<0.05)$ in exposed units for all CF bins except $0.14 \mathrm{kHz}$. (For parameters that relied on single exponential fits, the four histograms with double exponential adaptation functions were excluded from the analysis. $n \geq 78$ for each control scatter plot, $n \geq 64$ for each exposed scatter plot.) 


\section{Effects of acoustic overexposure}

The two most obvious effects of $48 \mathrm{~h}$ of intense sound exposure on single unit activity were drastically elevated $\mathrm{CF}$ thresholds and a reduction of spontaneous activity (Fig. 7). Threshold shifts were evident over almost the entire range of $\mathrm{CFs}$, except the lowest frequencies, and were greatest $(\sim 40 \mathrm{~dB})$ in the 1.14$\mathrm{kHz}$ analysis bin (see Methods for description of the CF bins). Likewise, there was a general reduction in spontaneous activity across CFs, with a near-complete loss in the analysis bin that had the greatest threshold increase. Both the threshold and spontaneous activity effects were similar to those previously reported for the same exposure conditions (Saunders et al. 1996b).

In spite of the dramatic changes in the spontaneous activity and sensitivity of exposed units, individual PST histograms were, for the most part, visually similar to controls. However, upon subjecting each parameter of the response to analysis, significant changes were found in exposed units. Averaged histograms suggested CF-specific differences in the adaptation functions as a consequence of exposure. Figure 8 shows the average exposed and control PST histogram for each of the analysis bins, which suggested that the onset response was larger, but the time constant was unaltered, in exposed units (data collected at the $\mathrm{CF}, 20 \mathrm{~dB}$ above threshold). These visual observations were confirmed upon statistical analysis. Peak firing rates were significantly elevated after the exposure in the $1.14-$ and $2.29-\mathrm{kHz}$ bins $(p<0.05$; Fig. $9 \mathrm{~A})$, whereas the adapted firing rates were unchanged $(p=0.609$, Fig. 9B). This indicates that, despite steeper rate-level function slopes after exposure (Saunders et al. 1996a, b; Plontke et al. 1999), the stimuli presented at $20 \mathrm{~dB}$ above threshold were inherently normalized with respect to the adapted firing rate, and the change in the response was more than just a simple scaling of the histogram envelope. The resulting adaptation functions in exposed ears, with their higher peak, but unchanged adapted, firing rates had increased

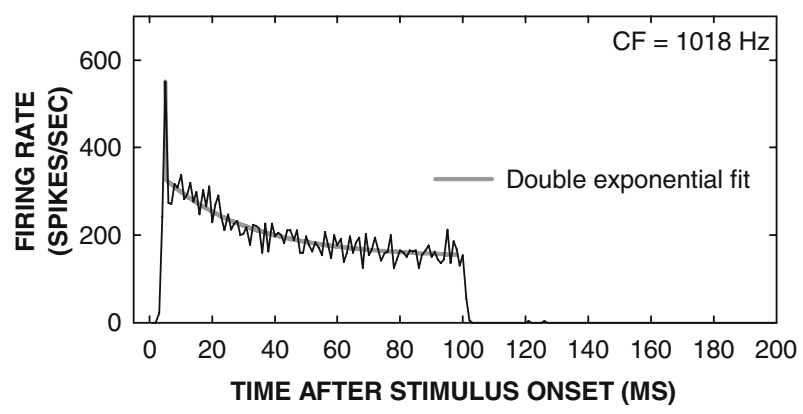

FIG. 10. Example of a histogram from an exposed unit with an adaptation function that was better described as a double exponential process. Three other exposed units showed similar behavior. percent adaptation $(p<0.05$ for the $0.57,1.14$ and $2.29 \mathrm{kHz}$ bins; Fig. 9D). The time constant with which the firing rate decayed was identical in exposed and control units $(p=0.982$, Fig. 9E). Even though the response decayed from an initially higher value with the same time constant, this short-term stage of adaptation went to completion in the 100 -ms period. This is shown by the near one-to-one relationship (linear regression slope $=0.95$ for both conditions) between the average spike rate occurring in the 91st through 100th 1-ms bins after stimulus onset and the final rate predicted for this stage by the exponential fits (Fig. 9C). For four exposed units, the adaptation function was better described as a double exponential, with a rapid adaptation component similar to that in Figure 5E. The response of one of these units is shown in Figure 10. Because the adapted firing rates and adaptation kinetics were largely unchanged across the population of units, the increased peak firing rate was associated with a larger phasic component in exposed units (Fig. 9F).

\section{DISCUSSION}

Adaptation across the tonotopic axis of the basilar papilla

The link between auditory nerve adaptation and hair cell synaptic processes has yet to be directly established, but a number of lines of evidence strongly point to the hair cell synapse as the source of adaptation. The amplitude of in vivo hair cell receptor potentials is constant during constant acoustic stimulation (Goodman et al. 1982). In addition, the $\mathrm{Ca}^{2+}$ currents that evoke neurotransmitter release display little inactivation over time in vitro (Zidanic and Fuchs 1995; Spassova et al. 2001). Thus, the cochlear mechanics and hair cell membrane events that occur before exocytosis appear to produce a constant output during constant stimulation. In spite of this, the rate of vesicle fusion decreases on a time scale similar to that of short-term adaptation (Moser and Beutner 2000; Spassova et al. 2004), and refilling of the emptied vesicle pool is kinetically similar to recovery from this adaptation (Spassova et al. 2004). Adaptation resulting from direct electrical stimulation of the auditory nerve (i.e., bypassing the hair cell) contrasts with acoustically evoked adaptation in that it is mild and seen only under certain conditions (Parkins 1989; Javel 1996; Miller et al. 2006). Miller et al. (2006) also note that adaptation to electric stimuli can be reduced by increasing the level of stimulation, a phenomenon not seen with acoustic stimulation. Furthermore, the insensitivity of chick short-term adaptation kinetics to temperature change is an argument against a role for neural refractoriness or 
receptor desensitization in adaptation (Crumling and Saunders 2005). Taken together, these observations support the notion that adaptation during acoustic stimulation arises from a decline in hair cell exocytosis due to the depletion of neurotransmitter vesicles. This interpretation is supported by the amplitude reduction of EPSPs seen in goldfish saccular afferents during sustained acoustic stimulation (Furukawa et al. 1978; Furukawa and Matsuura 1978).

The range of short-term adaptation time constants seen here $(5.7-55.9 \mathrm{~ms})$ is consistent with the depletion time of the chick hair cell readily releasable vesicle pool determined from patch-clamp recordings $(<50 \mathrm{~ms}$; Spassova et al. 2004). Units at high CFs had the shortest time constants, but the stimulus frequency manipulation data suggest that a large part of this variation with $\mathrm{CF}$ was caused by using different acoustic frequencies. The effect of the stimulus frequency on the time constant might be explained by events occurring in the hair cell. The receptor potentials of the hair cells driving the low CF units would be expected to follow to their lowfrequency stimulus tones much more than those in the hair cells driving the high CF units stimulated at high frequencies. With respect to the synapse, a hair cell receptor potential dominated by an AC component would likely be similar to a train of individual stimuli, with each acoustic cycle releasing a fractional portion of the available pool until it is depleted. As the driving frequency increases, the spacing between release events becomes smaller and, with a constant fraction of release, the pool depletes in a shorter time. At a high stimulation frequency, the hair cell receptor potential should be dominated by a DC component, producing a more constant stimulus for exocytosis, lasting for the duration of the acoustic signal. Under this condition, the dynamics of vesicle release would be governed by the intrinsic kinetics of exocytosis, similar to the situation in a depolarized voltage-clamped hair cell. Therefore, the time constant of $12.5 \pm 0.6 \mathrm{~ms}$ for the units in the highest CF bin $(2.28 \mathrm{kHz}$; Fig. $4 \mathrm{E})$, may be more indicative of the hair cell release kinetics, which have yet to be determined with sub-50 ms resolution in the chick.

Interestingly, the phasic component of the adapting response increased with CF (Fig. 4F) over papilla locations where dense body size and (presumably) the readily releasable pool increase systematically (Martinez-Dunst et al. 1997). The phasic component was resistant to changes in stimulus frequency, although peak and adapted rates increased in high CF units when stimulus frequency was lowered (Fig. 6). If phase locking of spikes to the stimulus frequency is a major contributor to firing rate, the spike rate should increase as the stimulus frequency rises, particularly in the low CF units. The lack of such an effect suggests that the CF gradient in peak firing rates could be attributable to a difference in the initial burst of exocytosis in hair cells at different papilla locations. This is what is predicted from the gradient in dense body size, where hair cells toward the more basal (high CF) end of the papilla have more capacity to hold vesicles at release sites. The distribution of phasic component sizes across CFs supports this notion, as long as the CF effect on the time constant has not prevented the short-term adaptation stage from completing before the end of the stimulus in low CF units, which appears to be the case (Fig. 4C). The magnitude of the phasic (adapting) response of the PST histograms increased over CFs up to $\sim 0.8 \mathrm{kHz}$. Dense body number in tall hair cells over the apical $2.0 \mathrm{~mm}$ of the basilar papilla remains constant, whereas the size increases substantially (Martinez-Dunst et al. 1997). Thus, the number of vesicles tethered to dense bodies increases over this range. Dense body number and size further than $2.0 \mathrm{~mm}$ from the apex (corresponding to a CF of $\sim 1.0 \mathrm{kHz}$; Manley et al. 1987; Chen et al. 1994) are unknown, but the phasic component data suggest that they may be constant toward the basal end of the cochlea.

The rate of vesicle fusion in chick tall hair cells is more than enough to support the firing rates measured in this report. At room temperature, a hair cell's readily releasable pool depletes its content of about 1000 vesicles in less than $50 \mathrm{~ms}$ (Spassova et al. 2004). This represents a fusion rate of least 20,000 vesicles/s in this initial burst of exocytosis. The reserve pool appears to be released at a constant rate of about 10,000 vesicles/s (Spassova et al. 2004). Because the typical tall hair cell divides its output between two synapses (Fischer 1992), the rate at which each afferent receives neurotransmitter is expected to be about half these values. Basal hair cells have seemingly more variability in their number of afferent contacts than apical hair cells (Fischer 1992), so this perhaps accounts for the increased scattering of firing rates and phasic component sizes in the high CF units (Fig. 4). Early evidence suggested that the neurotransmitter content of a single vesicle is sufficient to evoke an action potential in mammalian auditory afferents (Siegel 1992). In vitro recordings of excitatory postsynaptic currents (EPSCs) in rat auditory afferents support the idea that the postsynaptic potential induced by a single quantum is sufficient to bring an afferent to threshold (Glowatzki and Fuchs 2002). Yet amplitude histograms of the currents suggest a coordinated release mechanism, where the average EPSC results from the content of 3-6 vesicles. This would mean that many spikes are probably initiated by more than one vesicle. If this is also true 
in birds, the transmitter release rate seen by a typical afferent is still enough to produce the firing rates observed here. The readily releasable pool would be able to supply vesicles fast enough to achieve the $\sim 1000$ spikes/s maximum peak rate seen here, and the reserve pool could continue exocytosis at the rate needed to support the $\sim 400$ spikes/s maximum adapted rate (Fig. 5A and $\mathrm{B}$ ). In this scenario where the dynamics of release define the properties of adaptation, peak rate increases tonotopically because the readily releasable pool supplies vesicles at a higher rate in hair cells situated toward the basal end of the papilla; adapted rate is tonotopically constant because the reserve pool supplies vesicles at the same rate regardless of the hair cell's apical to basal position.

What is the utility of an adapting response in the primary auditory pathway? If the depletion model of adaptation is valid, it appears that the properties of hair cell exocytosis have evolved because they produce a beneficial burst of neural activity at stimulus onset. This increased probability that an action potential will be generated in the auditory nerve at the beginning of a stimulus might be important for certain perceptual tasks such as sound localization. Without the readily releasable pool, the neural encoding of stimulus onset might not be so precise and localization would suffer. Localization of low-frequency sounds would not be as dependent on an onset response, because auditory nerve spikes phase lock to these stimuli. This could be why low-frequency hair cells have small dense bodies and small adapting onset responses. With increase in $\mathrm{CF}$, phase information decreases whereas the onset response gets larger and faster, thereby maintaining a salient time stamp at the onset of a stimulus. The evidence of a cause-and-effect relationship between the readily releasable pool and short-term adaptation remains correlational, but the current study allowed comparison of hair cell synaptic anatomy and physiology to neural adaptation within the same species. The findings are consistent with a conclusion that shortterm adaptation and readily releasable pool depletion are related.

\section{Acoustic overexposure}

Anatomical evidence from alligator lizard hair cells suggested that dense body size and number and the number of tethered vesicles decrease after short acoustic overexposures that cause temporary threshold shifts (Henry and Mulroy 1995). Such a situation should decrease the initial burst of exocytosis and diminish the onset response in auditory neurons. In the overstimulated chick ear, however, the onset response was exaggerated after a 48-h exposure, suggesting that the readily releasable pool of vesicles was augmented, perhaps by an increase in the number of dense bodies and/or a greater number of vesicles tethered to dense bodies. Alternatively, the neurotransmitter content of individual vesicles may have increased. Regardless of the mechanism, increased neurotransmitter secretion at stimulus onset could cause the results presented in Fig. 9D and F, which resulted from the disparity in the effect on peak, vs. adapted, firing rates. It is difficult to imagine that heightened postsynaptic sensitivity could have caused the observed changes, because such a mechanism would likely have increased firing over the entire response. With the temperature insensitivity of adaptation kinetics (Crumling and Saunders 2005), receptor desensitization and afferent membrane properties are an unlikely source of adaptation in the normal ear, and it is doubtful that heightened postsynaptic sensitivity coupled with a new postsynaptic adaptation phenomenon would change the peak response without changing the adaptation time constant.

Along the lines of a change in time constant, four out of 68 exposed units showed a second adaptation time constant (see Fig. 10), something that was not observed in unexposed units under the same stimulus conditions. The effect of the exposure on the phasic component in the remaining 64 units was robust and occurred without a change in the time constant. One possible explanation for this emergent rapid component is that the hair cells were now releasing transmitter fast enough to drive the afferent up against its relative refractory period. Another possibility is that the dense body-associated vesicles had become so numerous in positions docked at the plasma membrane that they could be detected as a new kinetic component in the PST response. This would require that waiting, docked vesicles deplete in a kinetically different manner than the rest of the dense body vesicles. Activation of more vesicles from the existing docked population in the unit displaying rapid adaptation at $100 \mathrm{~dB}$ SPL (Fig. 5E) may likewise have caused the rapid component to emerge.

There is evidence for augmented vesicle pool size following strong stimulation in other systems. For example, in adrenal chromaffin cells, depolarization trains induce a $\mathrm{Ca}^{2+}$-dependent increase in the readily releasable pool (Smith et al. 1998). A similar conclusion of increased pool size was drawn from goldfish saccular afferent recordings to explain the effects of high $\mathrm{Ca}^{2+}$ perfusion on EPSPs (Furukawa and Matsuura 1978). Acoustic overstimulation produces an elevation in intracellular hair cell $\mathrm{Ca}^{2+}$ that lasts well beyond the end of the exposure (Fridberger et al. 1998) and may have initiated the changes in adaptation seen here. Whatever the mechanisms at play, it is fascinating to consider that the secure synaptic connection between the hair cell and its afferent responds to excessive stimulation with a form of 
synaptic facilitation. This may represent a compensatory response to diminished input from the transduction process during a period of induced hearing loss.

\section{ACKNOWLEDGMENTS}

This work was supported by NIH NIDCD grant R01DC00710 (JCS) as well as grants from the Pennsylvania Lions Hearing Research Foundation (JCS) and the National Organization for Hearing Research (MAC).

\section{REFERENCES}

Chen L, SAlvi R, Shero M. Cochlear frequency-place map in adult chickens: intracellular biocytin labeling. Hear. Res. 81:130-136, 1994.

Crumling MA, SAunders JC. Temperature insensitivity of short-term adaptation in single-units of the chick cochlear nerve. Synapse 58:243-248, 2005.

Eisen MD, Spassova M, Parsons TD. Large releasable pool of synaptic vesicles in chick cochlear hair cells. J. Neurophysiol. 91:2422-2428, 2004.

Fischer FP. Quantitative analysis of the innervation of the chicken basilar papilla. Hear. Res. 61:167-178, 1992.

Fridberger A, Flock A, Ulfendahl M, Flock B. Acoustic overstimulation increases outer hair cell $\mathrm{Ca}^{2+}$ concentrations and causes dynamic contractions of the hearing organ. Proc. Natl. Acad. Sci. USA. 95:7127-7132, 1998.

Furukawa T, Matsuura S. Adaptive rundown of excitatory postsynaptic potentials at synapses between hair cells and eight nerve fibres in the goldfish. J. Physiol. 276:193-209, 1978.

Furukawa T, HaYashida Y, Matsuura S. Quantal analysis of the size of excitatory postsynaptic potentials at synapses between hair cells and afferent nerve fibres in goldfish. J. Physiol. 276:211226, 1978.

Geisler CD, Greenberg S. A two-stage nonlinear cochlear model possesses automatic gain control. J. Acoust. Soc. Am. 80:13591363, 1986.

Glowatzki E, Fuchs PA. Transmitter release at the hair cell ribbon synapse. Nat. Neurosci. 5:147-154, 2002.

Goodman DA, Smith RL, Chamberlain SC. Intracellular and extracellular responses in the organ of Corti of the gerbil. Hear. Res. 7:161-169, 1982.

HaRris DM, Dallos P. Forward masking of auditory nerve fiber responses. J. Neurophysiol. 42:1083-1107, 1979.

HeNRY WR, Mulroy MJ. Afferent synaptic changes in auditory hair cells during noise-induced temporary threshold shift. Hear. Res. 84:81-90, 1995.

JAVEL E. Long-term adaptation in cat auditory-nerve fiber responses. J. Acoust. Soc. Am. 99:1040-1052, 1996.

Kaltenbach JA, Saunders JC. Spectral and temporal response patterns of single units in the chinchilla dorsal cochlear nucleus. Exp. Neurol. 96:406-419, 1987.

KIANG NYS. Discharge Patterns of Single Fibers in the Cat's Auditory Nerve. MIT Press, Cambridge, MA, 1965.
Lenzi D, Runyeon JW, Crum J, Ellisman MH, Roberts WM. Synaptic vesicle populations in saccular hair cells reconstructed by electron tomography. J. Neurosci. 19:119-132, 1999.

Manley GA, Brix J, Kaiser A. Developmental stability of the tonotopic organization of the chick's basilar papilla. Science 237:655-656, 1987.

Martinez-Dunst C, Michaels RL, Fuchs PA. Release sites and calcium channels in hair cells of the chick's cochlea. J. Neurosci. 17: 9133-9144, 1997.

Miller CA, Abbas PJ, Robinson BK, Nourski KV, Zhang F, Jeng FC. Electrical excitation of the acoustically sensitive auditory nerve: single-fiber responses to electric pulse trains. J. Assoc. Res. Otolaryngol. 7:195-210, 2006.

Moser T, Beutner D. Kinetics of exocytosis and endocytosis at the cochlear inner hair cell afferent synapse of the mouse. Proc. Natl. Acad. Sci. USA. 97:883-888, 2000.

PARKINS CW. Temporal response patterns of auditory nerve fibers to electrical stimulation in deafened squirrel monkeys. Hear. Res. 41:137-168, 1989.

PlontKe SK, Lifshitz J, Saunders JC. Distribution of rate-intensity function types in chick cochlear nerve after exposure to intense sound. Brain Res. 842:262-274, 1999.

Saunders JC, Doan De, Cohen Ye, Adler HJ, Poje CP. Recent observations on the recovery of structure and function in the sound-damaged chick ear. In: R.J S, Henderson D, Fiorino F, and Colletti V (eds) Auditory System Plasticity and Regeneration. Thieme Medical Publishers, New York, pp. 62-83, 1996a.

Saunders JC, Doan DE, Poje CP, Fisher KA. Cochlear nerve activity after intense sound exposure in neonatal chicks. J. Neurophysiol. 76:770-787, 1996b.

Schwid HA, GeIsler CD. Multiple reservoir model of neurotransmitter release by a cochlear inner hair cell. J. Acoust. Soc. Am. 72:1435-1440, 1982.

SIEgEL JH. Spontaneous synaptic potentials from afferent terminals in the guinea pig cochlea. Hear. Res. 59:85-92, 1992.

Smith RL, Brachman ML. Adaptation in auditory-nerve fibers: a revised model. Biol. Cybern. 44:107-120, 1982.

Smith CA, SJostrand FS. A synaptic structure in hair cells of the guinea pig cochlea. J. Ultrastruct. Res. 5:184-192, 1961.

Smith C, Moser T, Xu T, Neher E. Cytosolic $\mathrm{Ca}^{2+}$ acts by two separate pathways to modulate the supply of release-competent vesicles in chromaffin cells. Neuron 20:1243-1253, 1998.

Spassova M, Eisen MD, Saunders JC, Parsons TD. Chick cochlear hair cell exocytosis mediated by dihydropyridine-sensitive calcium channels. J. Physiol. 535:689-696, 2001.

Spassova MA, Avissar M, Furman aC, Crumling MA, Saunders JC, PARsons TD. Evidence that rapid vesicle replenishment of the synaptic ribbon mediates recovery from short-term adaptation at the hair cell afferent synapse. J. Assoc. Res. Otolaryngol. 5:376-390, 2004.

Westerman LA, Smith RL. Rapid and short-term adaptation in auditory nerve responses. Hear. Res. 15:249-260, 1984.

Westerman LA, Smith RL. Conservation of adapting components in auditory-nerve responses. J. Acoust. Soc. Am. 81:680-691, 1987.

Westerman LA, Smith RL. A diffusion model of the transient response of the cochlear inner hair cell synapse. J. Acoust. Soc. Am. 83:2266-2276, 1988.

ZiDANIC M, Fuchs PA. Kinetic analysis of barium currents in chick cochlear hair cells. Biophys. J. 68:1323-1336, 1995. 